\title{
Evaluation of Impact of Beam Size on Fibre Bragg Grating Fabrication
}

\author{
Adenowo A. Gbadebo, Elena G. Turitsyna and John A. R. Williams \\ Aston Institute of Photonics Technology, Aston University, Birmingham, B4 7ET \\ Author e-mail address: gbadebaa@aston.ac.uk
}

\begin{abstract}
We show the relationship between writing beam width and period chirp rate during fibre Bragg grating fabrication and the associated visibility constraints.

OCIS codes: (060.3735) Fiber Bragg gratings; (060.2300) Fiber measurements
\end{abstract}

\section{Introduction}

Fabrication of gratings has gone a long way since the onset by Kenneth Hill in 1976. Basic fabrication techniques such as holographic and phase-mask which have distinguishing advantages (variable wavelength, and high repeatability consecutively) have since been modified in an effort to combine the advantages of both methods [1]. These basic methods are inherently simple and have few controls, they have been combined and modified over time to enable the possibility of fabricating gratings with complex modulation index and phase profiles [1-3].

\section{Review}

Fabricating gratings with complex and precise profiles not only requires an elaborate equipment setup but a detailed look into the each of the components and trade-offs involved in the fabrication process. An ideal fabrication system would be one that can support a high range of phase change and modulation index change with high precision. Initial demonstrations used phase mask dithering to perform apodisation and phase change [4]. The phase mask dithering could only be run at low frequencies [5]. Amplitude modulators were later used which turned out to be power inefficient $[2,4]$. Phase modulators have also been used to extend the allowed phase range $[5,6]$ in order to increase the complexity in the profile written into a grating.

The number of fringes written has an impact on the amount of the total change possible per grating. This paper evaluates the effect of beam size and period change on the visibility of the grating written on the fibre. Generated grating profiles were used as an input to a high precision phase-mask fabrication setup. The experimental results were compared to the simulated grating.

To fabricate the gratings, the fibre was scanned across the phase mask based on the positioning and period. The beam was modulated based on the apodisation profile. Scanning of the beam across the fibre has the effect of writing multiple gratings on each other with a shift depending on the modulation and the speed of translation. This brings rise to a possibility of erasing the gratings already written.

The refractive index along the grating can be considered to be

$$
n(z)=n_{o}+\Delta n(z) \cos \left(\frac{2 \pi}{\Lambda(z)} z+\phi(z)\right),
$$

where $n_{o}$ is the effective refractive index across the fibre, $\Delta n(z)$ is the amplitude of index change in the grating, $\Lambda(z)$ is the period at position, $z$, along the grating; $\phi(z)$ is the change in phase along the grating. The maximum percentage change in period is proportional to the inverse of the number of fringes. The maximum period change erase a grating is, where $\Lambda_{\mathrm{B}}$ is the Bragg period and $w$, is the beam width. The visibility, $v(z)$, in amplitude index change can be related to the change in phase, $\phi(z)$, so that $v(z) \propto \cos (\phi(z))$. The visibility across the beam width can be expressed as

$$
v(z)=\frac{1}{w} \int_{-w / 2}^{w / 2} \cos \left(\frac{2 \pi \Delta \Lambda}{\Lambda_{B}^{2}} z\right) d z=\operatorname{sinc}\left(\pi w \Delta \Lambda / \Lambda_{B}^{2}\right)
$$

Here $\Delta \Lambda$ is the period change variation along the grating (constant over each fringe). The visibility is at maximum when $\Delta \Lambda=0$ i.e. there is no period change during writing. The simulated coupling coefficient, derived from the convolution of the desired apodisation and this visibility function, is used to simulate the transmission spectrum of the grating providing an estimation of the effect of the beam width on the final output.

\section{Results and discussion}

We considered a 21-channel comb filter grating and a 3-channel filter grating with equal spectral spacing. They were designed using the layer peeling (LP) algorithm [7], with the apodisation and period change shown in Fig. 1 and 2. In the case of 21-peaks grating, the downhill simplex optimisation algorithm was used for 
smoothing the apodisation profile. The gratings were fabricated with the system setup as in [8]. The measured beam size along the $\mathrm{x}$ axis during fabrication of 21-peaks grating was $400 \mu \mathrm{m}$. The reflection spectra of the fabricated gratings were measured with an Optical Vector Analyser and are shown in Fig.1b for the 21-peaks and Fig. $2 \mathrm{a}$ for the 3-peaks. The transmission spectra were simulated using the complex coupling coefficients of the gratings and the visibility function, $v(z)$. The produced profiles were compared with the measured reflection gratings. The measured and simulated data show a good match (Fig. 1b and Fig. 2a). One can see that due to a large beam width and complex period change in the case of 21-peaks grating, the reflection spectrum of the fabricated grating shows only 2 peaks instead of 21 as designed. However, it matches the calculated spectrum with applied visibility function (Fig. 1b).
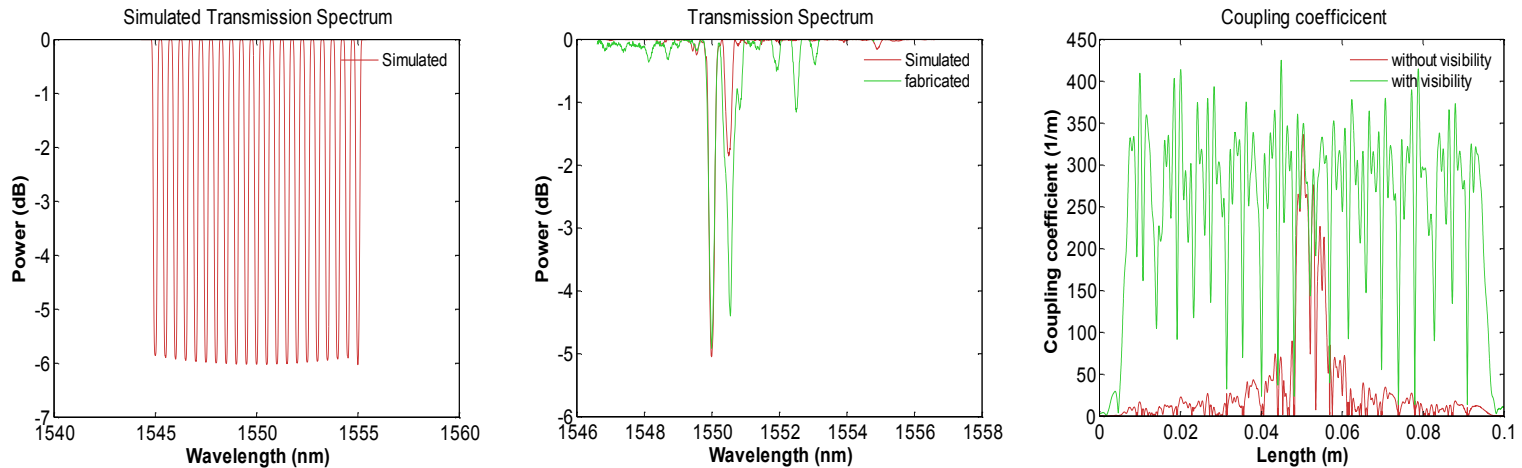

Fig.1. 21-peaks grating (a) Simulated transmission spectrum; (b) Fabricated transmission spectrum and simulated with visibility function.

(c) Coupling coefficient with and without visibility function
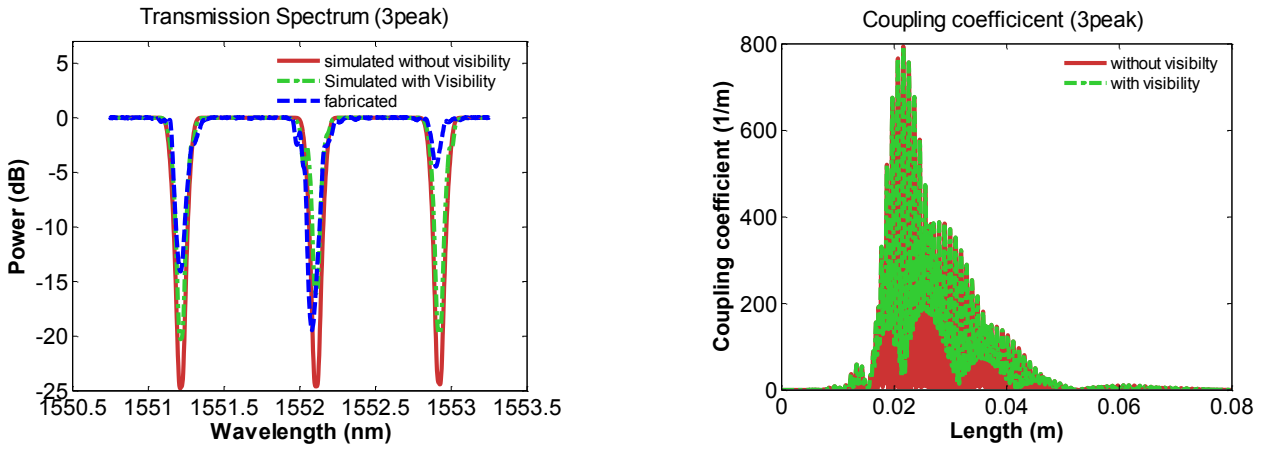

Fig.2. 3- peaks grating (a) Simulated transmission spectrum (red), fabricated transmission spectrum (blue) and simulated with visibility function (green) (b) Coupling coefficient with and without visibility function.

\section{Conclusion}

We show the importance of taking into account the beam width and period chirp rate of a grating during simulation and fabrication. It compares the fabrication of a grating with high period chirp rate and simulation of same grating with the visibility function applied. Further work is focused on compensating for loss in visibility due to high period change; also at using the visibility profile as a guide to generating profiles optimised with period change within the allowable region.

\section{References}

[1] R. Kashyap, Fiber bragg gratings, 2nd ed. (Academic Press, 2010).

[2] Y. Liu, J. Pan, and C. Gu, "Novel fiber Bragg grating fabrication method with high-precision phase control," Opt. Eng 43, 1916-1928 (2004).

[3] M. Gagné, L. Bojor, R. Maciejko, and R. Kashyap, "Novel custom fiber Bragg grating fabrication technique based on push-pull phase shifting interferometry.," Opt. Express 16, 21550-21557 (2008).

[4] J. Williams, "Method of fabricating an optical waveguide grating and apparatus for implementing the method," patent (2004).

[5] Y. Liu, "Advanced fibre gratings and their applications," Doctoral thesis, Aston University (2001).

[6] C. Sima, J. C. Gates, H. L. Rogers, P. L. Mennea, C. Holmes, M. N. Zervas, and P. G. R. Smith, "Ultra-wide detuning planar Bragg grating fabrication technique based on direct UV grating writing with electro-optic phase modulation.," Opt. Express 21, 15747-15754 (2013).

[7] Skaar, J., and Waagaard, O. H., "Design and characterization of finite-length fiber gratings," IEEE Journal of Quantum Electronics, 39 $1238-1245(2003)$

[8] A. Gbadebo, E. Turitsyna, J. Williams, and S. Turitsyn, "Fibre grating filters for suppression of near infrared OH emission lines," in Proceedings of 39th Eur. Conf. Exhib. Opt. Commun. (ECOC 2013), vol. 2013, no. 1, pp. 852-854, (2013). 